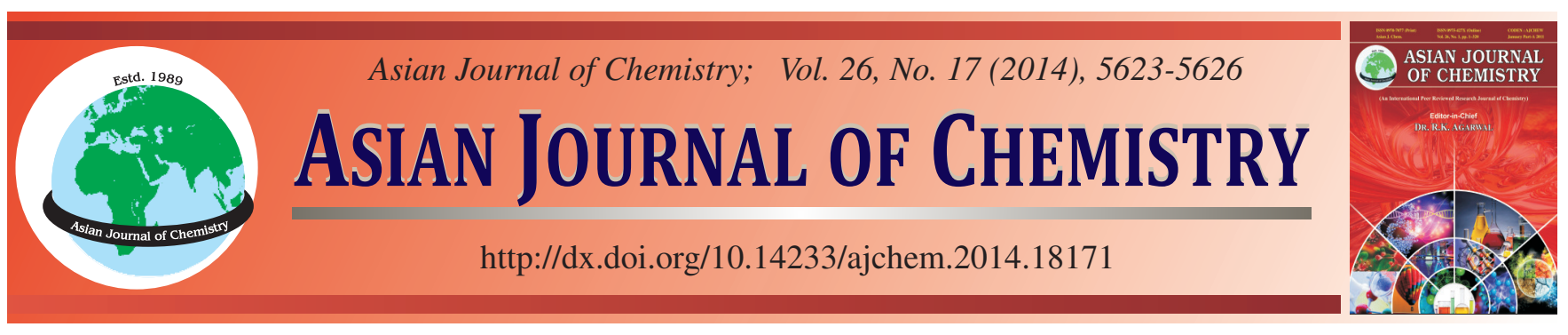

\title{
Reaction Mechanism for Selective Oxidation of Anethole to Anisaldehyde by Hydrogen Peroxide in Presence of Ferric Vanadate $\uparrow$
}

Xingdong Yao*, Caihua Peng and Zhanwang Shi

Key Laboratory of Forest Chemistry \& Engineering of Guangxi, School of Chemistry \& Chemical Engineering, Guangxi University for Nationalities, Nanning 530006, P.R. China

*Corresponding author: Tel/Fax: +86 771 3264360; E-mail: 13321716154@163.com

AJC-15760

\begin{abstract}
The reaction mechanism and kinetics for the oxidation of anethole to anisaldehyde by $\mathrm{H}_{2} \mathrm{O}_{2}$ in the presence of ferric vanadate have been investigated. The results show it is a homogeneous system with vanadium oxodiperoxo ion as the actual oxidant. The reaction follows the pseudo-first order rate equation. Free radical reaction mechanism has been verified by DPPH. A plausible reaction mechanism has been proposed based on the experimental results.
\end{abstract}

Keywords: Anethole, Anisaldehyde, Ferric vanadate, Hydrogen peroxide, Reaction mechanism.

\section{INTRODUCTION}

Anisaldehyde is an important perfume in fragrance and can also be used as an intermediate for synthesis of anisic acid, anisonitrile, porphyrins and other pharmaceutical products ${ }^{1,2}$. The conventional processes for the production of anisaldehyde employ $\mathrm{KMnO}_{4}, \mathrm{MnO}_{2}$ or $\mathrm{K}_{2} \mathrm{Cr}_{2} \mathrm{O}_{7}$ as oxidants ${ }^{3}$. Heavy metals in these oxidants and large amount of sulfuric acid used in the process may cause environmental problems. Many new processes have been developed for the production of anisaldehyde from anethole, including oxidation by ozone ${ }^{4}$, manganese dioxide $^{3}$, hydrogen peroxide ${ }^{5}, \mathrm{PhI}(\mathrm{OAc})_{2}{ }^{6}$ and electrochemical oxidation $^{3,7}$. Biological transformation ${ }^{8,9}$ has also been successfully applied for the synthesis of anisaldehyde. However, these processes still need to be improved for industrial application.

Hydrogen peroxide is a green oxidant with high content of active oxygen and water is the sole byproduct ${ }^{10}$. It has been applied for oxidation of unsaturated hydrocarbons to epoxides $^{11,12}$ and aldehydes ${ }^{13}$, alcohols to ketones and aldehydes to acids ${ }^{10}$. Application of the environmental friendly $\mathrm{H}_{2} \mathrm{O}_{2}$ is a hotspot for synthesis of anisaldehyde from anethole . $^{5}$

Vanadium is an effective component for many catalysts that can activate $\mathrm{H}_{2} \mathrm{O}_{2}$ and catalyze $\mathrm{H}_{2} \mathrm{O}_{2}$ oxidation of alcohols, olefins and their derivatives ${ }^{14,15}$. Vanadium(IV) complexes of aromatic carboxylic acids can effectively catalyze $\mathrm{H}_{2} \mathrm{O}_{2}$ oxidation of anethole to anisaldehyde with $100 \%$ conversion and
$73.5 \%$ selectivity for anisaldehyde ${ }^{5}$. We have reported that anethole can be selectively oxidized to anisaldehyde by $\mathrm{H}_{2} \mathrm{O}_{2}$ with a recyclable and reusable catalyst ferric vanadate $\left(\mathrm{FeVO}_{4}\right)^{16}$. The process was under mild conditions $\left(5 \mathrm{~h}\right.$ at $\left.40{ }^{\circ} \mathrm{C}\right)$ with $100 \%$ conversion for anethole and $73.4 \%$ selectivity for anisaldehyde. Investigation on the reaction mechanism would be helpful for the development and improvement of the anisaldehyde production process. In the present paper, the reaction kinetics and mechanism have been explored. The results indicate that it is a homogeneous catalytic system with free radical reaction mechanism and the high active diperoxyorthovanadate ion generated from $\mathrm{FeVO}_{4}$ and $\mathrm{H}_{2} \mathrm{O}_{2}{ }^{17}$ is the actual oxidant. Epoxide has been found as the reaction intermediate. The reaction follows pseudo-first order rate equation. A plausible reaction mechanism has been proposed based on the experimental results.

\section{EXPERIMENTAL}

Anethole, ferric nitrate nonahydrate, ammonium metavanadate, hydrogen peroxide (30\%), 1,4-dioxane, styrene and styrene epoxide are all AR grade from commercial and used without further purification. The $\mathrm{FeVO}_{4}$ catalyst was synthesized as previously reported ${ }^{17}$.

General procedure: A typical experiment for selective oxidation of anethole by $\mathrm{H}_{2} \mathrm{O}_{2}$ was performed as follows: anethole (0.4 g, $2.7 \mathrm{mmol}), \mathrm{FeVO}_{4}(0.004 \mathrm{~g}, 0.017 \mathrm{mmol})$, 
solvent $(15 \mathrm{~mL})$ and $30 \% \mathrm{H}_{2} \mathrm{O}_{2}(1.5 \mathrm{~mL}, 14.7 \mathrm{mmol})$ were mixed in a $100 \mathrm{~mL}$ round-bottomed flask equipped with magnetic stirrer and condenser. The mixture was stirred at $40{ }^{\circ} \mathrm{C}$ for $5 \mathrm{~h}$. After removal of the catalyst by centrifuge, the products in solution were analyzed by GC and/or GC-MS. To investigate oxidation of styrene or styrene epoxide for the reaction system, anethole was replaced with the same molar amount of styrene or styrene epoxide under the above reaction condition.

The oxidation products of anethole were analyzed on SP 6890 (Rainbow Chemical Instrument Co. Ltd., China) equipped with a SE-54 capillary column $(30 \mathrm{~m} \times 0.25 \mathrm{~mm} \times$ $0.25 \mu \mathrm{m}) . \mathrm{N}_{2}$ was used as the carrier gas with flow rate of 1.0 $\mathrm{mL} / \mathrm{min}$. The injector and FID detector were held at $250^{\circ} \mathrm{C}$. The oven was held at $160^{\circ} \mathrm{C}$. The injected volume was $1 \mu \mathrm{L}$. GC/MS analysis was performed on a Perkin Elmer Clarus 500 GC-MS instrument. Separation was achieved on ELite - 5MS capillary column $(30 \mathrm{~m} \times 0.25 \mathrm{~mm} \times 0.25 \mu \mathrm{m}$, Perkin Elmer, USA). The oven was held at $150{ }^{\circ} \mathrm{C}$ with injector and detector held at $250^{\circ} \mathrm{C}$. The Chemical composition and solubility of the catalyst in the reaction mixture were determined by ICPS6300 series ICP-AES spectrometer (ThermoFisher Scientific, USA). Cyclic voltammogram was measured on CHI760D electrochemical workstation (shanghai, China). The CV runs were recorded from $-1.6 \mathrm{~V}$ to $+1.5 \mathrm{Vrespective} \mathrm{to} \mathrm{Ag} / \mathrm{AgCl}$ as reference electrode at scan rate of $0.1 \mathrm{~V} / \mathrm{S}$ and the sensitivity $(\mathrm{A} / \mathrm{V})$ is $1 \mathrm{e}-5$.

The integration method was taken for kinetic measurement. General procedure is as follows: Anethole $(0.4 \mathrm{~g}, 2.7$ $\mathrm{mmol}), \mathrm{FeVO}_{4}(0.004 \mathrm{~g}, 0.017 \mathrm{mmol})$, solvent $(15 \mathrm{~mL})$ and $30 \% \mathrm{H}_{2} \mathrm{O}_{2}(1.5 \mathrm{~mL}, 14.7 \mathrm{mmol})$ were mixed in a $100 \mathrm{~mL}$ roundbottomed flask equipped with magnetic stirrer and condenser. The mixture was heated at 298, 303, 308 and $313 \mathrm{k}$. The concentration of anethole at different reaction times was determined by GC. The reaction order was found according to the linear relationship between $\ln \left(1 / \mathrm{C}_{\mathrm{A}}\right)-\mathrm{t}$ (first order reaction) or $1 / \mathrm{C}_{\mathrm{A}}-\mathrm{t}$ (second order reaction).

\section{RESULTS AND DISCUSSION}

It has been found that $\mathrm{H}_{2} \mathrm{O}_{2}$ cannot oxidize anethole directly in the absence of catalyst and $\mathrm{FeVO}_{4}$ could effectively catalyze the oxidation reaction, but amorphous $\mathrm{FeVO}_{4}$ demonstrated better catalytic performance than the catalyst with calcination. When the catalyst was mixed with $\mathrm{H}_{2} \mathrm{O}_{2}$ in dioxane, the supernatant became light yellow. If the supernatant was mixed with anethole, the conversion of anethole and the yield of anisaldehyde were the same as that in the presence of $\mathrm{FeVO}_{4}$. Therefore, this reaction system is in fact a homogeneous reaction.

It was well known that under neutral and basic conditions, vanadium(V) could form peroxovanadate complex with hydrogen peroxide via. 1-4 coordinated peroxide ligands. Under acidic conditions, addition of hydrogen peroxide to $\mathrm{VO}_{2}{ }^{+}$ gives the red oxomonoperoxo $\mathrm{VO}\left(\mathrm{O}_{2}\right)^{+}$and the yellow oxodiperoxo $\mathrm{VO}\left(\mathrm{O}_{2}\right)_{2}{ }^{-}$species ${ }^{18-21}$. $\mathrm{VO}\left(\mathrm{O}_{2}\right)_{2}{ }^{-}$is favored by low acid and high hydrogen peroxide concentrations ${ }^{22}$. The low acidic system ( $\mathrm{pH} \mathrm{3-5)} \mathrm{and} \mathrm{the} \mathrm{yellow} \mathrm{color} \mathrm{of} \mathrm{solution} \mathrm{for}$ the investigated catalytic system in the present paper indicated $\mathrm{VO}\left(\mathrm{O}_{2}\right)_{2}{ }^{-}$is the main specie of vanadium(V) in the reaction mixture, which was also verified by UV spectrometry. Since anethole cannot be oxidized by hydrogen peroxide directly, it is reasonable that $\operatorname{VO}\left(\mathrm{O}_{2}\right)_{2}{ }^{-}$is the actual oxidant. It can also be verified by cyclic voltammogram (Fig. 1). The reduction current increased from 0.3 to $0.8 \times 10^{-6} \mathrm{~A}$ after introduction of ammonium metavanadate or $\mathrm{FeVO}_{4}$ at $-0.5 \mathrm{~V}$, it is very clear the oxidation performance of $\mathrm{H}_{2} \mathrm{O}_{2}$ was significantly enhance in the presence of $\operatorname{vanadium}(\mathrm{V})$.

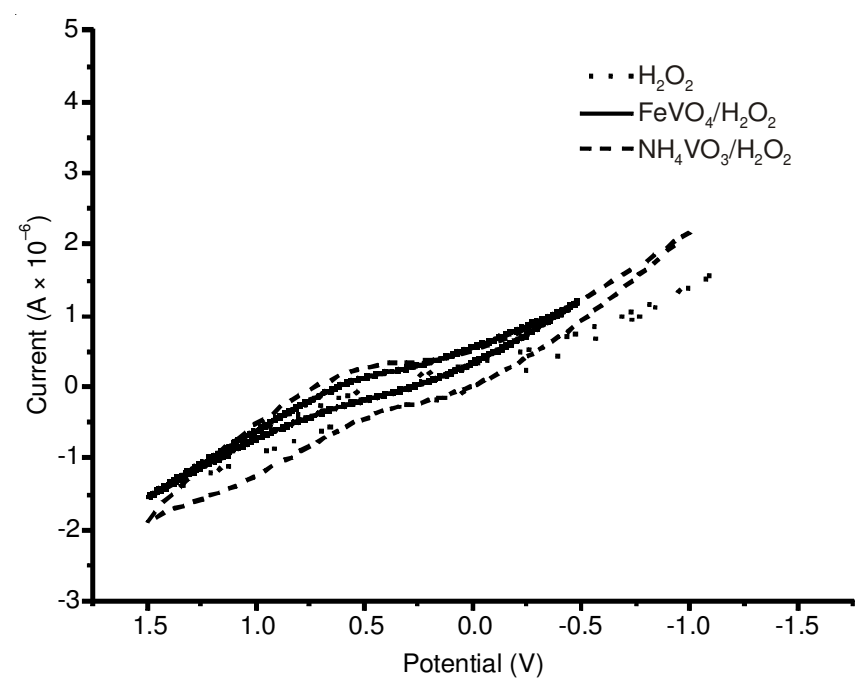

Fig. 1. Cyclic voltammogram for different systems. CV conditions: Init $\mathrm{E}(\mathrm{V})=-1.6$, High $\mathrm{E}(\mathrm{V})=1.5$, Low $\mathrm{E}(\mathrm{V})=-1.6$, Init $\mathrm{P} / \mathrm{N}=\mathrm{P}$, Scan Rate $(\mathrm{V} / \mathrm{S})=0.1$, Segment $=2$, Smpl Interval $(\mathrm{V})=0.001$, Quiet Time $=2$, Sensitivity $(A / V)=1 \mathrm{e}-5 . \mathrm{H}_{2} \mathrm{O}_{2}: 1.5 \mathrm{~mL} 30 \% \mathrm{H}_{2} \mathrm{O}_{2}$ in 15 $\mathrm{ml}$ dioxane; Ferric vanadate $/ \mathrm{H}_{2} \mathrm{O}_{2}: 0.017 \mathrm{mmol}$ ferric vanadate and $1.5 \mathrm{~mL} 30 \% \mathrm{H}_{2} \mathrm{O}_{2}$ in $15 \mathrm{~mL}$ dioxane; ammonium metavanadate/ $\mathrm{H}_{2} \mathrm{O}_{2}: 0.017$ mmol ammonium metavanadate and $1.5 \mathrm{~mL} 30 \% \mathrm{H}_{2} \mathrm{O}_{2}$ in $15 \mathrm{~mL}$ dioxane

As determined by ICP-AES spectrometry, the concentrations of vanadium and iron in the hydrogen peroxide/dioxane mixture were only 0.79 and $0.33 \mathrm{ppm}$, respectively. Therefore, the concentration of $\operatorname{VO}\left(\mathrm{O}_{2}\right)_{2}{ }^{-}$in the supernatant is very low, the high conversion of anethole indicates that it has very strong oxidation capacity for the oxidation of olefins. On the other hand, the decrease of catalytic performance for the catalyst after calcinations could be attribute to decrease of the solubility of the crystallized catalyst so as to lower the concentration of $\mathrm{VO}\left(\mathrm{O}_{2}\right)_{2}^{-}$.

While $0.023 \mathrm{mmol} \mathrm{NH}_{4} \mathrm{VO}_{3}, \mathrm{~V}_{2} \mathrm{O}_{5}$ and $\mathrm{NH}_{4} \mathrm{Fe}\left(\mathrm{SO}_{4}\right)_{2} \cdot 12 \mathrm{H}_{2} \mathrm{O}$ were employed as the catalysts, the conversion of anethole were $100,98.5$ and $18 \%$ and the corresponding selectivity for anisaldehyde were 70,54 and $61 \%$, respectively. The results indicated that vanadium $(\mathrm{V})$ is the main active component of the $\mathrm{FeVO}_{4}$ catalyst, but comparing with $\mathrm{NH}_{4} \mathrm{VO}_{3}$ and $\mathrm{V}_{2} \mathrm{O}_{5}$, the introduction of ferric ion has also contribution to improve the selectivity for anisaldehyde. This may be due to that high $\mathrm{VO}\left(\mathrm{O}_{2}\right)_{2}{ }^{-}$concentration which may cause over oxidation, which is unfavorable for high selectivity of anisaldehyde. $\mathrm{FeVO}_{4}$ has low solubility in the reaction mixture, thus the concentration of the oxodiperoxo complex in the mixture was very low so as to avoid over oxidation of anisaldehyde to anisic acid and other byproducts.

The effect of reaction time on the conversion of anethole and selectivity for anisaldehyde at $40^{\circ} \mathrm{C}$ was shown in Fig. 2. 


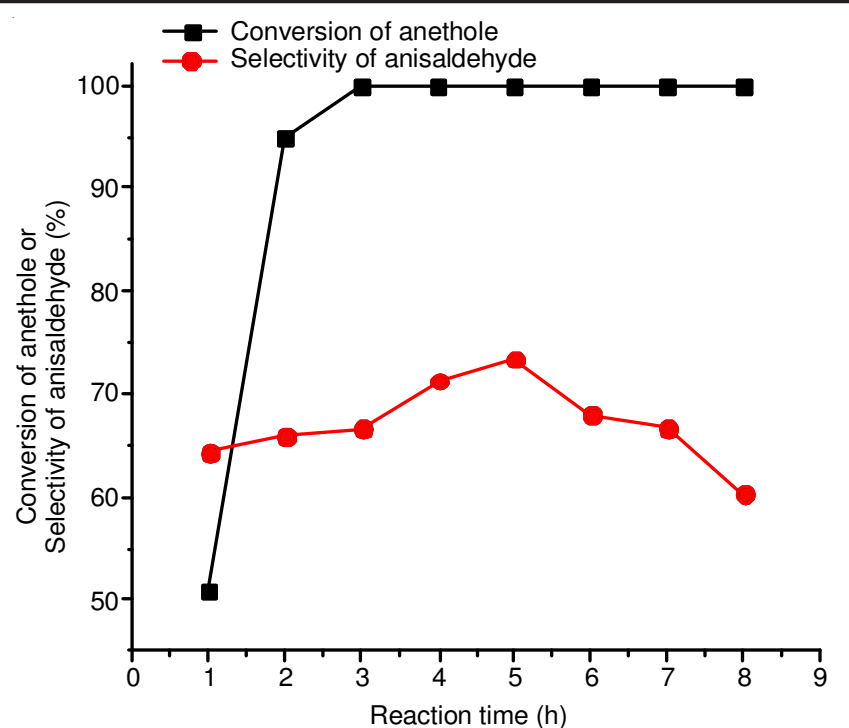

Fig. 2. Effect of reaction time on the conversion and selectivity at $40{ }^{\circ} \mathrm{C}$. Reaction conditions: 0.017 mmol $\mathrm{FeVO}_{4}, 2.7 \mathrm{mmol}$ anethole , 1.5 $\mathrm{mL} 30 \% \mathrm{H}_{2} \mathrm{O}_{2}$ in $15 \mathrm{~mL}$ dioxane

As shown in Fig. 2, anethole was almost completely converted in $2 \mathrm{~h}$, but the selectivity for anisaldehyde increased with prolonging reaction time and reached maximum in $5 \mathrm{~h}$. Then, the selectivity decreased gradually. These results indicated that a reaction intermediate was formed. It could be converted to anisaldehyde and the formed anisaldehyde could also be over oxidized to anisic acid so as to decrease its selectivity (the yield of anisic acid was 5.5 and $6.7 \%$, respectively after 6 and $7 \mathrm{~h}$ ).

The reaction mixture has been analyzed by GC/MS and it was found that anisaldehyde is the main product with anethole epoxide, anisic acid, p-methoxyphenylacetone and 1-(4methoxypheny)-2-hydroxy-propanone as the by-products. It has been reported that for most olefins oxidized by hydrogen peroxide, epoxide is the reaction intermediate ${ }^{23}$. To verify whether epoxide is the reaction intermediate for this oxidation reaction, anethole has been replaced with the same molar amount of styrene or styrene epoxide. It was found that benzaldehyde is the main product for styrene oxidation with small amount of 1-phenylethane-1,2-diol, 2-hydroxy-1-phenyl ethanone and benzoic acid in the reaction system. When the same molar amount of styrene epoxide was used to react with hydrogen peroxide under the same reaction conditions, similar result was found. Therefore, it is reasonable that epoxide is the reaction intermediate.

The $\mathrm{FeVO}_{4}$ promoted $\mathrm{H}_{2} \mathrm{O}_{2}$ oxidation system has been found effective for various substrates, but the structure of substrate demonstrates significant effect on the oxidation results. For example, when anethole, $\alpha$-methyl styrene, $p$-methylstyrene, styrene, 4-chlorostyrene and cinnamaldehyde have been used as substrates, the corresponding conversions were $100,62,55,39,34$ and $5 \%$, respectively. The results indicate that the oxidation reaction is an electrophilic process. High electron density of the double bond of substrate is beneficial for the oxidation reaction.

Electrophilic oxidation of olefins catalyzed by peroxides of transition metals (Mo, V, W, Ti etc.) has been reported in the following ways ${ }^{24-27}$ :

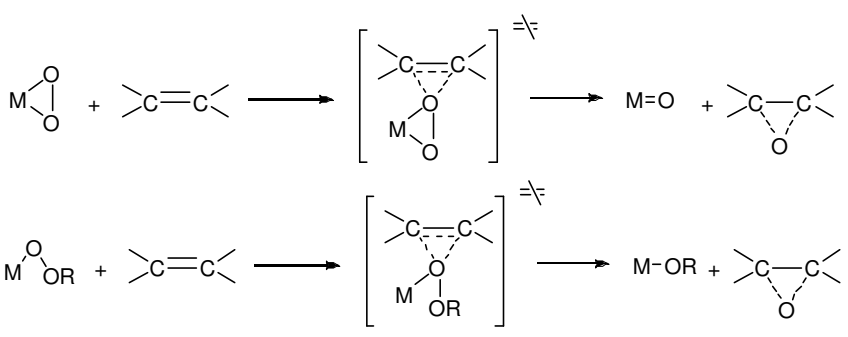

Usually these peroxides demonstrated good catalytic performance for such reactions. However, Mo, W and Ti compounds didn't demonstrate any catalytic effects for the oxidation of anethole by $\mathrm{H}_{2} \mathrm{O}_{2}$ as vanadium compounds. Therefore, the oxidation reaction may undergo in different way.

The oxodiperoxo complex has been reported as a versatile oxidant for oxidation and oxygenation reactions with radical or electrophilic mechanisms ${ }^{28}$. As discussed before, electrophilic oxidation couldn't explain the poor catalytic performance of $\mathrm{Mo}, \mathrm{W}$ and Ti compounds. To investigate the oxidation reaction mechanism, the free radical scavenger 2,2-diphenyl-1-picrylhydrazyl-hydrate (DPPH) has been employed. When 0.047 and $0.060 \mathrm{~g} \mathrm{DPPH}$ were added to the mixture, the corresponding conversion of anethole was 65 and $44 \%$, respectively. The dramatic decrease of conversion indicates it is a free radical reaction.

In order to investigate the catalytic nature of the present system, the reaction kinetics was also studied using the integration method. Good linear relationship was observed between $\ln \left(1 / \mathrm{C}_{\mathrm{A}}\right)-\mathrm{t}$, which indicated that the oxidation reaction follows the pseudo-first order rate equation. The rate constants at 298, 303,308 and $313 \mathrm{~K}$ were determined as $0.2451,0.6970,0.9700$ and $2.407 \mathrm{~h}^{-1}$, respectively. They were apparently increased with raising reaction temperature. The calculated activation energy $E_{a}$ from the slope of ln k-1/T was $107.37 \mathrm{KJ} / \mathrm{mol}$.

The oxidation of anethole by $\mathrm{H}_{2} \mathrm{O}_{2}$ in the presence of $\mathrm{FeVO}_{4}$ is in fact oxidation of anethole by $\mathrm{VO}\left(\mathrm{O}_{2}\right)_{2}{ }^{-}$to anethole epoxide first. Since the concentration of Vanadium in the supernatant is only $0.79 \mathrm{ppm}$, the concentration of $\mathrm{VO}\left(\mathrm{O}_{2}\right)_{2}{ }^{-}$is much lower than that of anethole. However, after the oxidation reaction, the reduced vanadium(IV) coordinate can be oxidized by excessive $\mathrm{H}_{2} \mathrm{O}_{2}$ to $\mathrm{VO}\left(\mathrm{O}_{2}\right)_{2}{ }^{-}$immediately, for the UV absorbance of solution at $220 \mathrm{~nm}$ for $\mathrm{VO}\left(\mathrm{O}_{2}\right)_{2}{ }^{-}$is almost constant during the oxidation process. Therefore, the concentration of oxidant $\mathrm{VO}\left(\mathrm{O}_{2}\right)_{2}{ }^{-}$could be regarded as constant during the oxidation process and the reaction rate can only depend on the concentration of anethole. This could be why the reaction follows the pseudo-first order rate equation.

Thus, a plausible mechanism was proposed for the oxidation of anethole by hydrogen peroxide in the presence of $\mathrm{FeVO}_{4}$ (Fig. 3).

\section{Conclusion}

The reaction kinetics and mechanism for catalytic oxidation of anethole by hydrogen peroxide in the presence of ferric vanadate under mild conditions $\left(5 \mathrm{~h}\right.$ at $\left.40{ }^{\circ} \mathrm{C}\right)$ has been investigated. The catalytic reaction is a homogeneous system and oxodiperoxo $\operatorname{VO}\left(\mathrm{O}_{2}\right)_{2}{ }^{-}$is the actual oxidant with free radical reaction mechanism. Epoxide has been found as the reaction intermediate. The reaction follows the pseudo-first order rate equation with the activation energy $E_{\mathrm{a}} 107.37 \mathrm{KJ} / \mathrm{mol}$ for the reaction. 


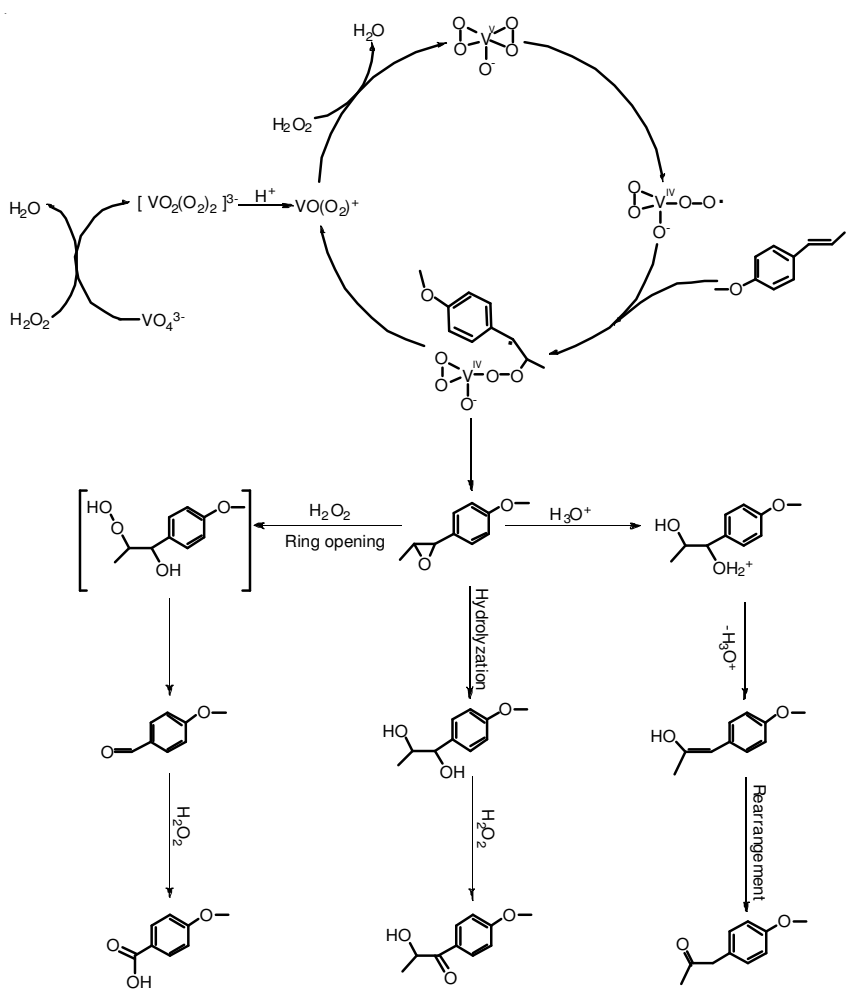

Fig. 3. Plausible reaction mechanism

\section{ACKNOWLEDGEMENTS}

The authors gratefully acknowledged the financial support for this study from Guangxi Natural Science Foundation (2011GXNS18014), Nanning Science and Technology Development Project (201109086G).

\section{REFERENCES}

1. Q. Wang, F. Liu and S. Yu, Adv. Fine Petrochem., 7, 34 (2006).

2. I. Kubo and I. Kinst-Hori, J. Agric. Food Chem., 46, 1268 (1998).

3. J. Grimshaw and C. Hua, Electrochim. Acta, 39, 497 (1994).

4. S. Fliszar and M. Granger, J. Am. Chem. Soc., 91, 3330 (1969).

5. Y. Xiao, H. Huang, D. Yin, D. Guo, L. Mao and Z. Fu, Catal. Commun., 10, 29 (2008).

6. H.M. Alvarez, D.P. Barbosa, A.T. Fricks, D.A.G. Aranda, R.H. Valdés and O.A.C. Antunes, Org. Process Res. Dev., 10, 941 (2006).

7. A.Y. Yang, S.R. Liu and G.M. Cao, Syn. Chem., 3, 147 (1995).

8. K. Okamoto, S. Narayama, A. Katsuo, I. Shigematsu and H. Yanase, J. Biosci. Bioeng., 93, 207 (2002).

9. H. Mang, J. Gross, M. Lara, C. Goessler, H. Schoemaker, G.M. Guebitz and W. Kroutil, Tetrahedron, 63, 3350 (2007).

10. R. Noyori, M. Aoki and K. Sato, Chem. Commun., 16, 1977 (2003).

11. N. Mizuno, K. Yamaguchi and K. Kamata, Coord. Chem. Rev., 249, 1944 (2005).

12. K. Kamata, K. Yonehara and Y. Sumida, Science, 300, 964 (2003).

13. P. Wright and J. Abbot, Int. J. Chem. Kinet., 25, 901 (1993).

14. G.B. Shul'pin, Y. Ishii, S. Sakaguchi and T. Iwahama, Russ. Chem. Bull., 48, 887 (1999).

15. V. Conte, F. Di Furia and G. Licini, Appl. Catal. A, 157, 335 (1997).

16. X. Yao, C. Peng, Y. Nie, Z. Shi and F. Lei, Chinese Patent 201310180914.6 (2013).

17. G. Kakabadse and H.J. Wilson, Nature, 180, 861 (1957).

18. O.W. Howarth and J.R. Hunt, J. Chem. Soc., Dalton Trans., 1388 (1979).

19. A.T. Harrison and O.W. Howarth, J. Chem. Soc., Dalton Trans., 1173 (1985).

20. N.J. Campbell, A.C. Dengel and W.P. Griffith, Polyhedron, 8, 1379 (1989).

21. F. Secco, Inorg. Chem., 19, 2722 (1980).

22. M.J. Clague and A. Butler, J. Am. Chem. Soc., 117, 3475 (1995).

23. Y. Ishii, K. Yamawaki, T. Ura, H. Yamada, T. Yoshida and M. Ogawa, J. Org. Chem., 53, 3587 (1988).

24. G. Strukul, Catalytic Oxidations with Hydrogen Peroxide as Oxidant Kluwer Academic, Dordrecht, p. 177 (1992).

25. A.G.J. Ligtenbarg, R. Hage and B.L. Feringa, Coord. Chem. Rev., 237, 89 (2003).

26. C. Bolm, Coord. Chem. Rev., 237, 245 (2003).

27. J. Hartung and M. Greb, J. Organomet. Chem., 661, 67 (2002).

28. A. Butler, M.J. Clague and G.E. Meister, Chem. Rev., 94, 625 (1994). 